\title{
Realization of a micrometre-sized stochastic
}

\section{heat engine}

\author{
Valentin Blickle ${ }^{1,2 \star}$ and Clemens Bechinger ${ }^{1,2}$
}

The conversion of energy into mechanical work is essential for almost any industrial process. The original description of classical heat engines by Sadi Carnot in 1824 has largely shaped our understanding of work and heat exchange during macroscopic thermodynamic processes'. Equipped with our present-day ability to design and control mechanical devices at micro- and nanometre length scales, we are now in a position to explore the limitations of classical thermodynamics, arising on scales for which thermal fluctuations are important ${ }^{2-5}$. Here we demonstrate the experimental realization of a microscopic heat engine, comprising a single colloidal particle subject to a time-dependent optical laser trap. The work associated with the system is a fluctuating quantity, and depends strongly on the cycle duration time, $\tau$, which in turn determines the efficiency of our heat engine. Our experiments offer a rare insight into the conversion of thermal to mechanical energy on a microscopic level, and pave the way for the design of future micromechanical machines.

Macroscopic heat engines operating periodically between two heat baths are described well by the laws of thermodynamics, owing to the large number of internal degrees of freedom, which render fluctuations negligible. In contrast, fluctuations become visible when the typical energy scales of engines are reduced by more than twenty orders of magnitude, down to values around $k_{\mathrm{B}} T$. This regime can be achieved when the typical system dimensions are scaled down from metres to micrometres ${ }^{2}$. Such conditions are typically met for biomolecules ${ }^{6,7}$ and other microelectromechanical systems (MEMS; refs 8-10) that can perform translational or rotational motion as a result of chemical reactions and electrical fields. Also, several types of Brownian motor have been discussed that are able to extract useful work by rectification of thermal noise $\mathrm{s}^{11-13}$. Despite its conceptual simplicity, no attempt has been made to realize a microscopic heat engine that extracts energy by cyclically working between two heat baths in a regime where fluctuations dominate.

Here we present an experimental realization of a Stirling engine, where a single Brownian particle is subjected to a time-dependent optical trap and periodically coupled to different heat baths ${ }^{14}$. The particle and the trapping potential replace the working gas and the piston of its macroscopic counterpart. As for conventional heat engines where the periodic coupling of the working gas to a cold and a hot heat bath changes volume and pressure inside a piston, the fluctuations of a colloidal particle subjected to an external optical potential vary on changing the temperature of the solvent.

As a Brownian particle we used a single melamine bead of diameter $2.94 \mu \mathrm{m}$ suspended in water and confined in a vitreous sample cell $4 \mu \mathrm{m}$ in height. Using a highly focused infrared laser beam we exerted a parabolic trapping potential $U(R, k)=$ $(1 / 2) k(t) R^{2}$ on the particle, where $R$ is its radial distance from the trapping centre and $k(t)$, the time-dependent trap stiffness. The value of $k$ is determined by the laser intensity, which is controlled by an acousto-optic modulator. By means of video microscopy, the two-dimensional trajectory was sampled with spatial and temporal resolutions of $10 \mathrm{~nm}$ and $33 \mathrm{~ms}$, respectively. Keeping hot and cold reservoirs thermally isolated at small length scales is experimentally very difficult to achieve, so rather than coupling our colloidal particle periodically to different heat baths, here we suddenly changed the temperature of the surrounding liquid.

This variation of the bath temperature was achieved by a second coaxially aligned laser beam whose wavelength was matched to an absorption peak of water. This allowed us to heat the suspension from room temperature to $90^{\circ} \mathrm{C}$ in less than $10 \mathrm{~ms}$ (ref. 15). The temperature inside the cell was measured by means of a temperature sensitive dye. (For experimental details, see Methods.)

Figure 1 shows the comparison of a macroscopic Stirling cycle and its realization in our microscopic experiment. The cycle starts at time $t=0$ with step (1), where the particle is trapped at room temperature $T_{\mathrm{c}}=22^{\circ} \mathrm{C}$ (corresponding to the cold heat bath) in a shallow potential with $k_{\min }=281 \mathrm{fN} \mu \mathrm{m}^{-1}$. In analogy to a macroscopic engine (inset Fig. 1), for $0 \leq t<\tau / 2$ we perform an isothermal compression by linearly increasing the trap stiffness

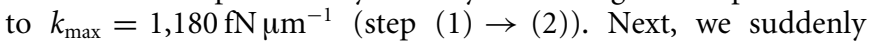
$(<1 \mathrm{~ms})$ turn on the heating laser with a mechanical shutter, which leads (relative to our sampling frequency) to an instantaneous temperature jump to $T_{\mathrm{h}}=86^{\circ} \mathrm{C}$. Because the trapping potential remains constant during the step $(2) \rightarrow(3)$, it corresponds to an isochoric process. Next, the system is isothermally expanded during $\tau / 2 \leq t<\tau$, corresponding to $(3) \rightarrow(4)$, and the entire cycle of total duration $\tau$ is completed with a temperature jump back to $T_{\mathrm{c}}(\operatorname{step}(4) \rightarrow(1))$.

To extract thermodynamic quantities from the particle's positional fluctuations we use the framework of stochastic thermodynamics. For an overdamped particle, as considered here, the increment of work $\mathrm{d} W=(\partial U(R, t) / \partial t) \mathrm{d} t$ is directly related to the particle's trajectory ${ }^{16}$. With the sign convention used here, work is negative (positive) when energy is extracted (delivered) from (to) the colloidal system. Similarly, the increment of heat $\mathrm{d} Q=(\partial U(R, t) / \partial R) \dot{R} \mathrm{~d} t$ is positive when the heat is transferred from the particle to the thermal bath. Integration along the stochastic trajectory yields the time-dependent work

$$
W(t)=\int_{0}^{t} \frac{\partial U\left(R\left(t^{\prime}\right), t^{\prime}\right)}{\partial t^{\prime}} \mathrm{d} t^{\prime}
$$

From equation (1) it is obvious that-in agreement with a macroscopic Stirling engine-the isochoric transitions in the microscopic Stirling engine $((2) \rightarrow(3),(4) \rightarrow(1)$ in Fig. 1$)$ do

\footnotetext{
12nd Physical Institute, Physics Department, University of Stuttgart, D-70569 Stuttgart, Germany, ${ }^{2}$ Max Planck Institute for Intelligent Systems, D-70569 Stuttgart, Germany. *e-mail: v.blickle@physik.uni-stuttgart.de.
} 


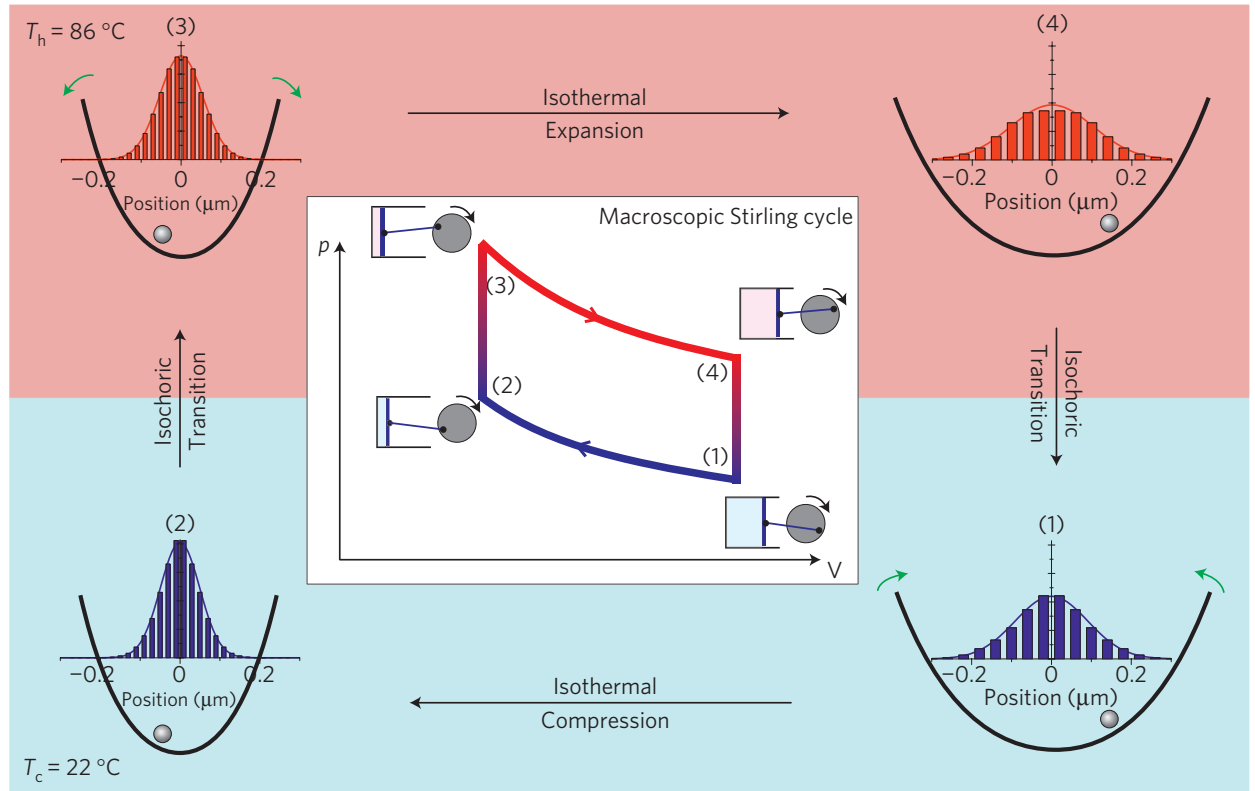

Figure 1 | Schematic comparison of a macroscopic Stirling cycle and its realization in a colloidal system. (1) $\rightarrow$ (2) Isothermal increase of the stiffness of the optical trapping potential (isothermal compression) at $T_{\mathrm{C}}=22^{\circ} \mathrm{C}$. (2) $\rightarrow$ (3) Instantaneous temperature increase to $T_{\mathrm{h}}=86^{\circ} \mathrm{C}$ at fixed optical potential (isochoric process). (3) $\rightarrow$ (4) Isothermal decrease of trap stiffness (isothermal expansion) at $T_{\mathrm{h}}$. (4) $\rightarrow$ (1) Instantaneous temperature decrease to $T_{\mathrm{c}}$ at fixed optical potential (isochoric process). The histograms show the measured particle probability distributions of the corresponding stationary states together with Gaussian fits as solid lines. The width of the distribution at $T_{\mathrm{h}}$ is 1.11 times greater than at $T_{\mathrm{c}}$ at same potential. Originating in an increase in thermal energy this broadening provides an independent measure of the hot temperature $\left(90^{\circ} \mathrm{C}\right)$ which is in good agreement with the fluorescently measured temperature of $86^{\circ} \mathrm{C}$. Inset Stirling process in the pressure-volume diagram representation, where the enclosed area amounts to the work extracted by the machine.

not contribute to $W$ because $U$ is constant and the work is only delivered during the isothermal steps. Although work $W$, heat $Q$ and inner energy $U$ are no longer sharp values but have become fluctuating quantities, energy can be neither generated nor destroyed, which leads to a stochastic first-law-like energy balance $\Delta U=W-Q$ (refs 16,17).

Figure 2a shows how $W$ (in units of $k_{\mathrm{B}} T_{\mathrm{c}}$ ) changes with time during the Stirling cycle (black) over the duration of a typical experiment. As the slope of the work trajectory is negative, the machine extracts energy on the order of $20 k_{\mathrm{B}} T \cong 10^{-19} \mathrm{~J}$ from the heat baths. To resolve the dynamics within a cycle, we show a magnified view in the inset of Fig. 2a. Obviously, $W$ decreases (increases) when expanding (compressing) the system because the corresponding work increments $\mathrm{d} W=(\partial U / \partial t) \mathrm{d} t$ are always negative (positive). In contrast to a macroscopic engine, however, here thermal fluctuations in the work are obvious. These fluctuations can be seen more clearly in Fig. 2b,c where we show the work per cycle $n, w_{n}=\int_{n \tau}^{(n+1) \tau}\left(\partial U / \partial t^{\prime}\right) \mathrm{d} t^{\prime}$ and its probability distribution $p\left(w_{n}\right)$, which is centred around its average value $\bar{w}=-0.11 k_{\mathrm{B}} T_{\mathrm{c}}$. We also performed experiments where the Stirling engine was operated in counter-clockwise direction. This is achieved by time reversal of the protocol that controls both the optical tweezer and the heating laser. As expected, the slope of $W$ then becomes positive and the machine operates as a heat pump (red data in Fig. 2).

For a microscopic understanding of our stochastic heat engine it is helpful to consider that the work increment $\delta W$ of a colloidal particle at position $R$ inside a parabolic trap with time-dependent trap stiffness is proportional to $R^{2}$. The fact that the particle probability distribution at higher temperatures is broader explains the observed small difference between the work extracted during step $(3) \rightarrow(4)$ and the work spent while compressing the system (1) $\rightarrow(2)$. Although this broadening is hardly visible in the corresponding equilibrium distributions
(Fig. 1), it results in an average work production. For the counterclockwise direction, this difference is positive, as is the slope of the work trajectory.

We also studied how the average work per cycle $\bar{w}$ varies with cycle time $\tau$ (red symbols in Fig. 3a). With increasing $\tau, \bar{w}$ monotonically decreases until it approaches the quasistatic limit $\bar{w}_{\infty}$ at long cycle times. In this limit, the work is easily obtained from the corresponding free energies along the paths $(1) \rightarrow(2)$ and $(3) \rightarrow(4)$ of the Stirling cycle. Because the free energy of a colloidal particle inside a two-dimensional parabolic potential is (up to a constant) given by $F(k, T)=2 k_{\mathrm{B}} T \ln \sqrt{k}$ (ref. 18), we obtain

$$
\bar{w}_{\infty}=\Delta F_{1 \rightarrow 2}+\Delta F_{3 \rightarrow 4}=2 k_{\mathrm{B}} T_{\mathrm{c}}\left[1-\frac{T_{\mathrm{h}}}{T_{\mathrm{c}}}\right] \ln \sqrt{\frac{k_{\max }}{k_{\min }}}
$$

which agrees well with our experimental data (Fig. 3a). Towards smaller cycle times, we observe a reversal from negative to positive values of $\bar{w}$. In this regime, dissipation effects become important and the mean work per cycle can be written as

$$
\bar{w}=\bar{w}_{\infty}+\bar{w}_{\text {diss }}
$$

with $\bar{w}_{\text {diss }}$ the mean irreversibly dissipated work per cycle. Because $\bar{w}_{\text {diss }}>0$ and increases with decreasing $\tau$, this qualitatively explains the observed change of sign in $\bar{w}$. It has been shown ${ }^{14,18,19}$ that the irreversible work can be written to first order as $\bar{w}_{\text {diss }}=\Sigma / \tau$, where the coefficient $\Sigma$ contains information about the protocol and the coupling mechanism between the colloidal particle and the thermal environment. We find excellent agreement between equation (2) (solid line) and our data when the fitting parameter $\Sigma$ is adjusted to $\Sigma=0.95 k_{\mathrm{B}} T_{\mathrm{c}} \mathrm{s}$.

For most practical applications the power $P=-\bar{w} / \tau$ delivered by a micro-engine is of central importance. In contrast to macroscopic heat machines, where the dependence of $P$ on the cycle 
a

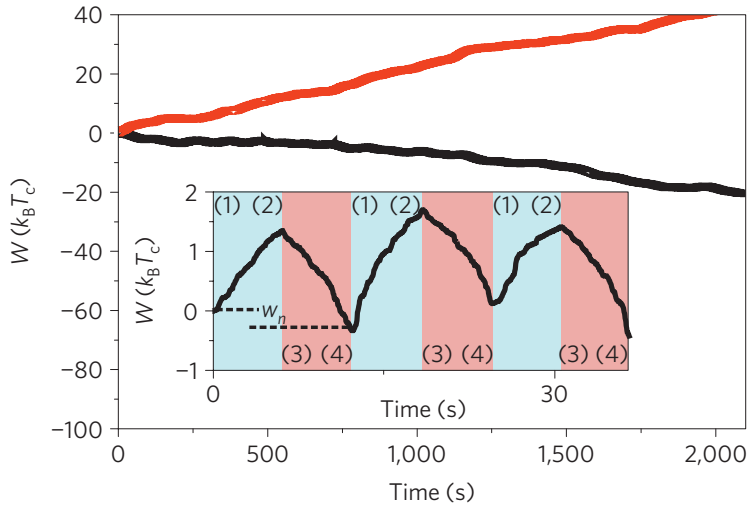

b
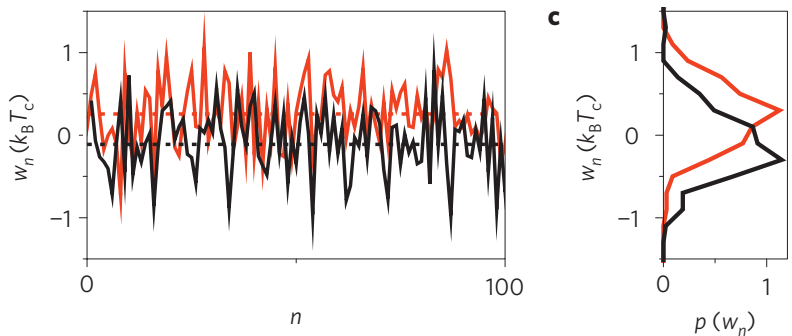

Figure 2 | Extracted work of the microscopic heat engine. a, $W$ versus time for a heat engine in clockwise (black curve) and counter-clockwise (red curve) cycle direction with $\tau=12 \mathrm{~s}$. In the latter case, the machine acts as a heat pump. (Inset) Magnified view where the individual steps during three cycles are resolved. $\mathbf{b}$, Work accumulated over one cycle as a function of the cycle number $n$ for clockwise (black) and counter-clockwise (red) cycle directions. The average work per cycle is $-0.11 \mathrm{k}_{\mathrm{B}} T$ and $0.25 \mathrm{k}_{\mathrm{B}} T$, respectively, for the two cases. c, Corresponding probability distributions, $p\left(w_{n}\right)$, to the plots in $\mathbf{b}$

time was discussed in 1975 by Curzon and Ahlborn ${ }^{20}$, only recently has this dependence been theoretically investigated for microscopic heat engines ${ }^{14,19}$. To achieve the maximum power output of a heat machine there is an obvious trade-off between large cycling times where dissipation becomes negligible and $\bar{w}$ is large, and small cycle times where the Stirling engine operates at high frequencies. Indeed, such a maximum of the mean power is found in our experiments around $\tau=7 \mathrm{~s}$ (black symbols in Fig. 3a).

Finally, we also investigated the efficiency of our engine, which is given by the ratio between extracted work and the average absorbed heat $\bar{q}_{\mathrm{h}}$ at the hot temperature, that is $\eta=\bar{w} / \bar{q}_{\mathrm{h}}$. Using the first law of thermodynamics it follows that the heat absorbed during the expansion at $T_{\mathrm{h}}$ is $\bar{q}_{3 \rightarrow 4}=\bar{w}_{3 \rightarrow 4}-\Delta \bar{u}_{3 \rightarrow 4}$. In thermal equilibrium, that is, in the quasistatic limit $\tau \rightarrow \infty$, the equipartition theorem holds and the average inner energy at each position in the protocol is equal to $k_{\mathrm{B}} T$. Therefore $\Delta \bar{u}_{3 \rightarrow 4}=0$ and thus $\bar{q}_{3 \rightarrow 4}=\bar{w}_{3 \rightarrow 4}$. To perform step (3) $\rightarrow$ (4) quasistatically the system has to relax into equilibrium after the sudden temperature increase $(2) \rightarrow$ (3). This relaxation is connected to an additional average heat flow $\bar{q}_{2 \rightarrow 3}=-\Delta \bar{u}_{2 \rightarrow 3}=-k_{\mathrm{B}} T_{\mathrm{h}}\left(1-T_{\mathrm{h}} / T_{\mathrm{c}}\right)$. With $\bar{w}_{3 \rightarrow 4}=\Delta F_{3 \rightarrow 4}$, the efficiency becomes

$$
\eta_{\infty}=\bar{w}_{\infty} /\left(\Delta F_{3 \rightarrow 4}-\Delta \bar{u}_{2 \rightarrow 3}\right)=\eta_{\mathrm{c}}\left[1+\eta_{\mathrm{c}} / \ln \left(k_{\max } / k_{\min }\right)\right]^{-1}
$$

where $\eta_{\mathrm{c}}=1-T_{\mathrm{c}} / T_{\mathrm{h}}$ is the Carnot efficiency. It should be mentioned that an identical expression is obtained for a macroscopic Stirling engine ${ }^{21}$ when $k_{\max } / k_{\min }$ is replaced by the compression ratio. From the experimental values given in the caption of Fig. 3, we obtain $\eta_{\infty}=0.14=0.9 \eta_{\mathrm{c}}$.

As shown in Fig. 3b, the experimentally determined $\eta$ indeed converges to $\eta_{\infty}=0.14$ at large $\tau$. As a result of dissipation

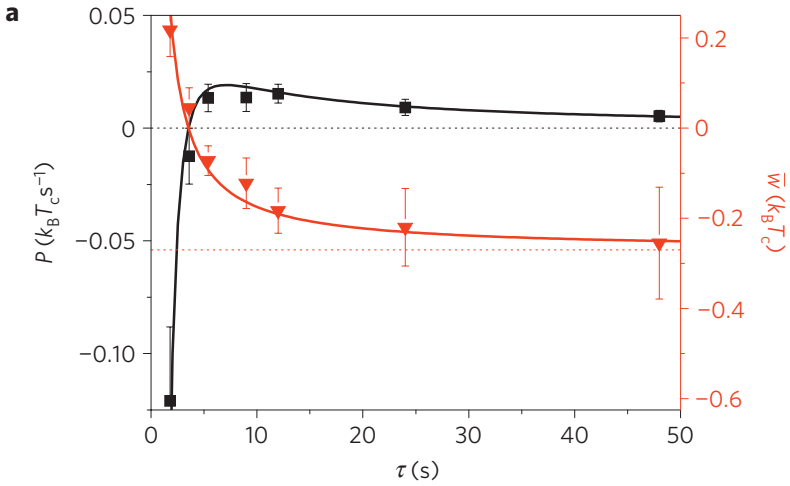

b

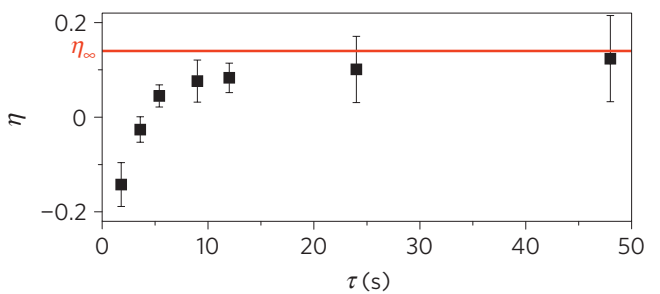

Figure 3 | Power, work and efficiency versus the cycle revolution time $\boldsymbol{\tau}$. a, Averaged work $\bar{w}$ and power as a function of the cycle time $\tau$ for the experimental conditions $T_{\mathrm{h}}=76^{\circ} \mathrm{C}, T_{\mathrm{C}}=22^{\circ} \mathrm{C}, k_{\max } / k_{\min }=4.4$, $\bar{w}_{\infty}=-0.27 k_{\mathrm{B}} T_{\mathrm{c}}$ and $\eta_{\mathrm{c}}=0.15$. The red solid line is a fit according to $\bar{w}=\bar{w}_{\infty}-\Sigma / \tau$ with the free fitting parameter $\Sigma=0.95 k_{B} T_{c} s$. For $\tau<3.6 \mathrm{~s}$ the machine is not extracting energy, $\bar{W}$ becomes positive and the output power negative. $\mathbf{b}$, Efficiency $\eta$ versus cycle time. In the long-time limit the measured efficiency approaches $\eta_{\infty}=0.14$ (solid line). Note that the efficiency at maximum power is $\eta=0.08$. The error bars indicate the standard deviations of the mean among individual cycles.

effects, the efficiency becomes negative, meaning the colloidal particle is not able to perform work below $\tau<t_{\mathrm{s}}=3.6 \mathrm{~s}$ and the machine stalls ${ }^{14}$. At maximum power $(\tau=7.2 \mathrm{~s})$ we obtain $\eta_{\max }=0.075$, which is, within our experimental error, in agreement with the Curzon ${ }^{20}$ prediction of $\eta_{\max }=1-\sqrt{T_{\mathrm{c}} / T_{\mathrm{h}}}=0.081$. It should be mentioned that recent theoretical work has demonstrated considerable deviations from this prediction for $\left(T_{\mathrm{h}}-T_{\mathrm{c}}\right) / T_{\mathrm{c}} \gg 1$ (refs 14,19). In our aqueous experiment the maximal temperature difference is restricted by the melting and freezing temperatures of water and therefore $\left(T_{\mathrm{h}}-T_{\mathrm{c}}\right) / T_{\mathrm{c}}$ does not exceed 0.3 . Thus, the aforementioned deviations are negligible.

Depending on their range of use, future micromechanical devices will be operated either at highest efficiency or maximum power. As shown above, these values are determined by the amount of irreversible work, which depends on $\tau$. However, they are also influenced by the specific choice of the protocol. Because the bath temperature and trap stiffness are externally controlled in our set-up, this allows the realization of arbitrarily protocols (and even Carnot cycles). It will be interesting to identify the protocol under which the maximum power is optimized. Furthermore, a feedback based on the instantaneous particle position could be added to the protocol. To what extent this will affect the maximum power remains an open question.

\section{Methods}

Particle trapping and tracking. All experiments were performed using melamine particles of diameter $2.94 \mu \mathrm{m}$ (Microparticles, MF-F-S1712). A highly diluted aqueous suspension was confined in a thin, $4 \mu \mathrm{m}$ high, measurement chamber. The custom-built cell consisted of two BK7 glass slides, pressed together in the presence of polystyrene spacer particles $4 \mu \mathrm{m}$ in diameter (Duke, 4204A) and then sealed with epoxy glue. To create the time-dependent harmonic trapping potential, a laser beam ( $\lambda=1,064 \mathrm{~nm}$, Coherent Compass) was sent through an acousto-optic modulator (Crystal Technology, AOM 3080) and focussed to a diffraction limited 
spot, with a maximal intensity of $20 \mathrm{~mW}$, using a $\times 10$ microscope objective (Zeiss Epiplan, NA 0.2). The particle was imaged on the chip of a charge-coupled device camera (Basler, scout scA640) using a custom-built microscope with a $\times 50$ objective (Leica PL Fluotar). Its position was detected by means of a standard video microscopy routine (real-time threshold tracking using LabView). The sampling frequency, the magnification ( $180 \mathrm{~nm}$ per pixel) and the detected pixels (about 300) determine the temporal/spatial resolution of the experimental set-up as being $10 \mathrm{~nm} / 33 \mathrm{~ms}$ (ref. 22).

Laser heating and temperature control. For optical heating we used a second coaxially aligned laser (IPG Raman Fiber Laser, $5 \mathrm{~W})$, with its wavelength $(\lambda=1,455 \mathrm{~nm})$ tuned to an absorption peak of water. At this wavelength the absorptivity of water is more than 200 times greater than at $\lambda=1,064 \mathrm{~nm}$, leading to an absorption length of only $300 \mu \mathrm{m}$. To avoid temperature gradients on the length scale of our field of view the spot size of the heating laser was set to $70 \mu \mathrm{m}$. The temperature inside the cell was measured by means of a Rhodamine dye (TAMRA, $70 \mathrm{umoll}^{-1}$ ), dissolved in Tris-HCL buffer solution $\left(0.1 \mathrm{moll}^{-1}\right)$. As the $\mathrm{pH}$ of the Tris-HCL solution depend on the temperature and the quantum yield (measured with a Nikon G2-A filter set) of the dye varies with $\mathrm{pH}$, from the measured fluorescent intensity distribution the local temperature can be determined with an accuracy of $\pm 2{ }^{\circ} \mathrm{C}$ (ref. 23).

Working in a thin measurement cell has several advantages. First, the absorption length in water of the heating laser radiation $(300 \mu \mathrm{m})$ is large compared with the cell thickness, thus the heating along the axial direction is homogeneous. Second, as only a very thin layer of water is heated the system's thermal relaxation time is fast ( $<10 \mathrm{~ms}$; ref. 15). Last, convectional forces, which decrease quadratically with the cell thickness ${ }^{24}$, are negligible.

Received 13 July 2011; accepted 8 November 2011; published online 11 December 2011

\section{References}

1. Carnot, S. Reflexions sur la Puissance Motorice Du Feu et Sur Les Machines (Ecole Polytechnique, 1824).

2. Bustamante, C., Liphardt, J. \& Ritort, F. The nonequilibrium thermodynamics of small systems. Phys. Today 58, 43-48 (July, 2005).

3. Seifert, U. Stochastic thermodynamics: Principles and perspectives. Eur. Phys. J. B 64, 423-431 (2008).

4. Jarzynski, C. Equalities and inequalities: Irreversibility and the second law of thermodynamics at the nanoscale. Annu. Rev. Condens. Mater. P 2 329-351 (2011).

5. Campisi, M., Hanggi, P. \& Talkner, P. Colloquium: Quantum fluctuation relations: Foundations and applications. Rev. Mod. Phys. 83, 771-791 (2011).

6. Svoboda, K., Schmidt, C. F., Schnapp, B. J. \& Block, S. M. Direct observation of kinesin stepping by optical trapping interferometry. Nature 365, 721-727 (1993).

7. Noji, H., Yasuda, R., Yoshida, M. \& Kinosita, K. Direct observation of the rotation of $\mathrm{F}_{1}$ - ATPase. Nature 386, 299-302 (1997).

8. Barreiro, A. et al. Subnanometer motion of cargoes driven by thermal gradients along carbon nanotubes. Science 320, 775-778 (2008).

9. Fennimore, A. M. et al. Rotational actuators based on carbon nanotubes. Nature 424, 408-410 (2003).
10. Steeneken, P. G. et al. Piezoresistive heat engine and refrigerator. Nature Phys. 7, 354-359 (2011).

11. Reimann, P. Brownian motors: Noisy transport far from equilibrium. Phys. Rep. 361, 57-265 (2002).

12. Hanggi, P. \& Marchesoni, F. Artificial Brownian motors: Controlling transport on the nanoscale. Rev. Mod. Phys. 81, 387-442 (2009).

13. Toyabe, S., Muneyuki, E., Sagawa, T., Ueda, M. \& Sano, M. Experimental demonstration of information-to-energy conversion and validation of the generalized Jarzynski equality. Nature Phys. 6, 988-992 (2010).

14. Schmiedl, T. \& Seifert, U. Efficiency at maximum power: An analytically solvable model for stochastic heat engines. Epl-Europhys. Lett. 81, 20003 (2008).

15. Cordero, M. L., Verneuil, E., Gallaire, F. \& Baroud, C. N. Time-resolved temperature rise in a thin liquid film due to laser absorption. Phys. Rev. E 79, 011201 (2009).

16. Sekimoto, K. Langevin equation and thermodynamics. Prog. Theor. Phys. Suppl. 130, 17-27 (1998).

17. Blickle, V., Speck, T., Helden, L., Seifert, U. \& Bechinger, C. Thermodynamics of a colloidal particle in a time-dependent nonharmonic potential. Phys. Rev. Lett. 96, 070603 (2006).

18. Sekimoto, K. Stochastic Energetics (Springer, 2010).

19. Esposito, M., Kawai, R., Lindenberg, K. \& Van den Broeck, C. Efficiency at maximum power of low-dissipation Carnot engines. Phys. Rev. Lett. 105, 150603 (2010).

20. Curzon, F. L. \& Ahlborn, B. Efficiency of a Carnot engine at maximum power output. Am. J. Phys. 43, 22-24 (1975).

21. Wongwises, S. \& Kongtragool, B. A review of solar-powered Stirling engines and low temperature differential Stirling engines. Renew. Sust. Energy Rev. 7, 131-154 (2003).

22. Crocker, J. C. \& Grier, D. G. Methods of digital video microscopy for colloidal studies. J. Colloid Interface Sci. 179, 298-310 (1996).

23. Duhr, S., Arduini, S. \& Braun, D. Thermophoresis of DNA determined by microfluidic fluorescence. Eur. Phys. J. E 15, 277-286 (2004).

24. Rusconi, R., Isa, L. \& Piazza, R. Thermal-lensing measurement of particle thermophoresis in aqueous dispersions. J. Opt. Soc. Am. B 21, 605-616 (2004).

\section{Acknowledgements}

We thank J. Mehl and U. Seifert for inspiring discussions and critical reading of the manuscript. We are grateful to D. Braun for providing us with helpful information regarding the fluorescence temperature detection and the IR heating of water. V.B. was supported by the Deutsche Forschungsgesellschaft (Grant No. BL 1067).

\section{Author contributions}

V.B. designed the experiment, carried out the experiments, analysed the data and wrote the manuscript. C.B. designed the experiment and wrote the manuscript.

\section{Additional information}

The authors declare no competing financial interests. Reprints and permission information is available online at http://www.nature.com/reprints. Correspondence and requests for materials should be addressed to V.B. 